\title{
An Algorithm for Autonomous Isolation of Neurons in Extracellular Recordings*
}

\author{
Edward A. Branchaud and Joel W. Burdick \\ Mechanical Engineering \\ California Institute of Technology \\ Pasadena, CA 91125 \\ eddie@caltech.edu
}

\author{
Richard A. Andersen \\ Dept. of Biology \\ California Institute of Technology \\ Pasadena, CA 91125
}

\begin{abstract}
This paper describes novel multi-electrode systems that can autonomously position recording electrodes inside cortical tissue so as to isolate and then maintain optimal extracellular signal recording quality without human intervention. Autonomous microdrives can be used to improve the quality and efficiency of acute recordings that are needed for basic research in neurophysiology. They also offer the potential to increase the longevity and quality of chronic recordings and will serve as the front end of neuroprosthetic systems that aid the handicapped. We first describe the autonomous positioning algorithm, and its implementation as a finite state machine. We have deployed the algorithm on both conventional acute recording micro-drives and a novel miniature robot microdrive. Experimental results in monkey cortex are presented.
\end{abstract}

Index Terms-Brain-Machine Interface, Neural Recording, Control Algorithm

\section{INTRODUCTION}

This paper describes an algorithm for autonomously positioning extracellular neural recording electrodes to achieve optimal signal quality. This algorithm is the heart of a control system that supervises a new generation of novel miniature robots that autonomously establish and optimize the interface between electrodes and neurons. A companion paper [1] describes these novel miniature robotic devices.

As described in more detail below, establishing and maintaining a high quality electrode-neuron interface is an essential component for future generations of neural prosthetic systems that can aid the handicapped. Such autonomous control algorithms, coupled with robotic recording devices, can also enable simultaneous unsupervised recordings of multiple neurons in acute preparations, which are vital to basic research in neuroscience.

Previously [2], [3], we described an elementary algorithm for autonomously positioning electrodes to isolate neurons. In this paper we describe a more robust algorithm accounting for the biophysical challenges of neuron isolation, a user-friendly software version available to the neuroscience community, and results of the implementation of the algorithm in several prototypical acute recording setups. Before describing the algorithm and our experimental results, we first briefly review the current practice

\footnotetext{
${ }^{*}$ This work has been supported by NIH and DARPA
}

of extracellular recording of neurons and the potential contribution of the described algorithm. We then describe the essential components of the algorithm, and experimental results obtained by deploying the algorithm on a number of different electro-mechanical platforms.

\section{Current Issues in Extracellular ReCORDing}

Information transfer and processing in the brain occurs through the transmission of electrical pulses, called action potentials, between neurons. Studying the patterns of action potentials associated with individual neurons while a subject (e.g. a rat, fly, monkey, or human) is presented with a stimulus or engages in a behavioral task is a principal tool for studying brain areas. Noninvasive methods such as fMRI or EEG recordings can provide gross estimates of activity levels in a given region, but recording action potentials of individual neurons is necessary to understand how information is processed in local neural networks. Recordings of action potentials are made by inserting electrodes (typically sharpened metal wires insulated along their length and exposed at the tip) into the neural tissue.

Because action potentials emitted by a neuron are highly stereotyped in shape and information is encoded in their timing, a successful extracellular recording is one in which the firing of action potentials of individual neurons can reliably be detected; the neurons are then considered "isolated". Fig. 1 shows an example action potential recording with one isolated neuron and a second neuron with action potentials that cannot reliably be separated from fluctuations due to noise.

There are two dominant modes of recordings. In acute recordings, electrodes are inserted and removed from the neural tissue each recording session. In chronic recordings, electrodes are surgically implanted and remain in place for weeks, months, or possibly years at a time. Both practices will benefit from the ability to autonomously isolate a neuron and then maintain optimal isolation in the presence of perturbations.

\section{A. Acute Recordings}

For acute recordings, a portion of the skull over the brain region of interest is removed and replaced with a sealable chamber. During a recording session, a device termed a microdrive is affixed to the opened chamber and 

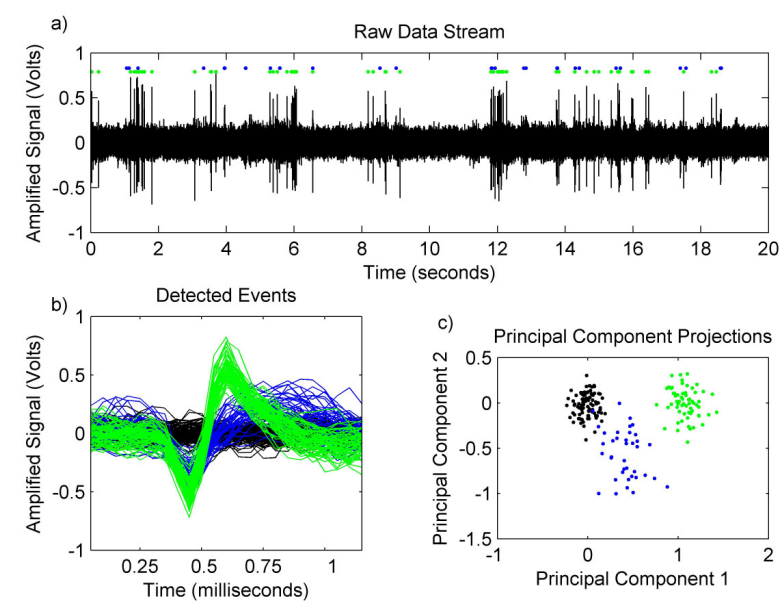

C) Principal Component Projections

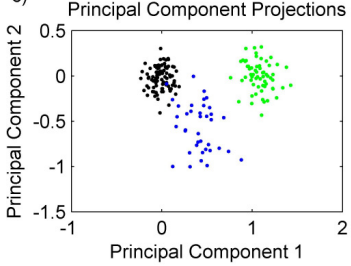

Fig. 1. Example of neuron isolation. a) Filtered (High-pass 10Hz) signal. The dots show detected events. b) Detected events aligned by their minimum. The black traces are random samples of noise. The events have been categorized as originating from two neurons. c) Projections of detected events and noise samples on the first two principal components. The neuron firing the events shown in blue is not isolated, as its action potentials could be confused with noise fluctuations. The other neuron (in green) is far from the noise, but is not very well isolated as its action potentials could be confused with those in blue.

is used to advance the electrodes into the neural tissue, usually in a motorized fashion. The electrode motion is manually controlled by the experimenter until a neuron is sufficiently isolated. This process is commonly guided by experience, intuition and feedback from visual and auditory representations of the voltage signal to the experimenter. The electrode is positioned close enough to the neuron for a high quality recording, yet far enough away to avoid damaging the neuron. During the course of a typical experiment, the electrode must be periodically repositioned by the operator to maintain a quality signal. Repositioning is necessary due to tissue migration and tissue decompression that occurs naturally. The process of isolating and maintaining neural signals consumes a significant amount of the experimenter's time and focus.

Simultaneous recordings with many electrodes are becoming an increasingly important technique for understanding how local networks of neurons process information, as well as how brain areas communicate with each other. Commercial microdrives (i.e., motorized electrodes) with sixteen or more electrodes are currently available [4]. As the number of electrodes increases, the task of positioning each electrode to maintain a high quality neural signal becomes intractable for a single experimenter to manage. Data collection in these experiments is currently limited by how many channels an experimenter can effectively monitor. An algorithm for automating this process will significantly improve the efficiency and quality of multielectrode studies.

\section{B. Chronic Recordings}

In chronic recordings, stationary multi-electrode assemblies, which are typically bundles or arrays of thin wires or silicon probes, are surgically implanted in the region of interest [5]-[7]. The signal yield of the implant array, i.e. the percentage of the array's electrodes that record active cells, depends upon the luck of the initial surgical placement. The electrodes may be placed in inactive tissue or the wrong brain region. Even if properly placed, the active recording site may not sit sufficiently close to an active cell body (the electrically active tip of a recording electrode must lie within 40-60 microns of the neuron's soma to provide a useful signal [8]). Moreover, even if the electrode is initially well placed, tissue migrations (due to blood pressure variations, breathing, and mechanical shocks) and local tissue reactions can cause subsequent loss of signal, thereby reducing or disabling the function of the recording array over time.

Chronically implanted recording arrays are the essential front end for future neural prosthetic systems that are aimed at aiding the severely handicapped. Clearly, longevity of the implanted electrode arrays is necessary, as repeated and frequent surgical intervention to implant new electrodes is not desirable. In previous papers [2], [3], [9], we described initial steps towards a chronic multi-electrode implant in which the electrodes can be continually and autonomously repositioned after implantation. Such an implant could overcome the above limitations and greatly extend the signal yield and lifetime of chronic array implants. A companion paper [1] in this conference describes a novel miniature robotic multi-electrode system, which illustrates the kinds of systems upon which we envision the deployment of this algorithm. In order to be useful in a clinical application, such as using neural recordings as control signals for an external device (i.e. neural prosthetics), the electrodes in a chronic repositionable array will have to be autonomously controlled to maintain optimal signal recording quality. This will require an algorithm like the one described in this paper, coupled with miniaturization via MEMS-fabrication [9].

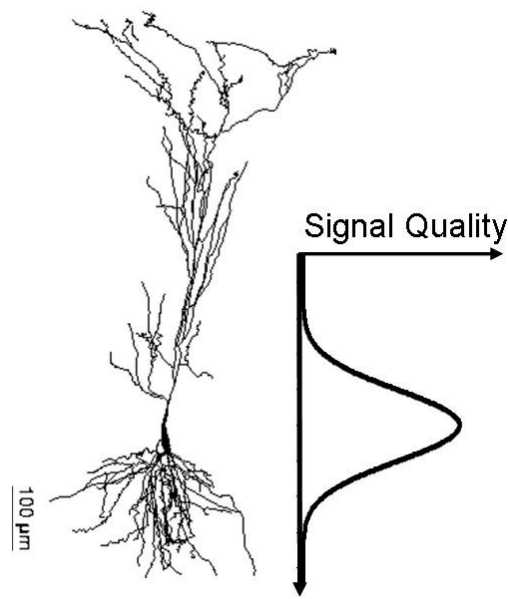

Fig. 2. Illustration of rat pyramidal neuron based on morphology in [11]. The overlaid curve illustrates the idealized signal quality observed by an electrode as it passes the action potential initiation location on the neuron. 


\section{Algorithm ReQuirements}

In order to isolate neurons, experimenters perform a number of difficult tasks, including event detection (detecting the presence and onset of an action potential), unsupervised classification of neural signals (i.e. classifying neural events without a priori knowledge of their number or structure), and accounting for stochastic neuron activity and complex mechanical interactions between the electrode and the neural tissue. What follows is a brief listing of the major challenges faced in automating the isolation process.

1) Unsupervised Detection, Classification and Data Associtation: Action potentials of varying amplitudes and shapes must be autonomously detected and grouped by the neuron from which they originate. In conventional practices, this process is normally performed by an experimenter who manually sets thresholds and identifies distinguishing signal features. The data association problem is also faced when attempting to track the signals arising from distinct neurons while moving the electrode.

2) Variable Firing Rates: A general procedure for autonomously isolating a neuron involves sampling the amplitude of action potentials at several locations and searching for the local maximum of the signal quality. Depending on the behavioral state of the recording subject, the neuron which is being isolated may stop firing action potentials for one or more sampling periods, leading to false estimates of the signal amplitude at those locations. The algorithm must be robust to these false estimates.

3) Tissue Relaxation: During the initial insertion of the electrodes, neural tissue is compressed, and the subsequent decompression over the course of the experiment causes the neurons to drift relative to the electrode. Optimal recording positions are moving targets. It is quite common that action potentials will be observed for some time and then disappear; presumably the neuron has either drifted out of range or stopped firing. Also, once a neuron is isolated, the electrode must be periodically readjusted to maintain the isolation. Often, neurons drift away from the line of travel of the electrode and become impossible to isolate. An effective algorithm must account for this drift when isolating and maintaining isolations and must recognize the appearance and disappearance of isolatable neurons from its line of travel.

4) Local Electrode-Tissue Interactions (Hysteresis): In addition to the gross tissue relaxation occurring over the several hours of the experiment, there can exist local mechanical coupling between the electrode tip and the neural tissue, causing hysteresis in the signal (i.e. commanding a move forward and then backward by the same amount does not yield the same signal). We postulate two possible causes of this hysteresis. There may be stiction between the electrode tip and the tissue. Additionally, because of tissue compression from the electrode insertion, when the electrode moves backward, the tissue may relax with it, resulting in a smaller relative movement between the electrode tip and the tissue than expected. This hysteresis is highly variable in magnitude, limits control action and adds uncertainty in the electrode placement.

5) Neuron Damage: The electrode can potentially puncture and damage neurons. This can happen while the electrode moves to achieve isolation, or when tissue relaxes towards a stationary electrode. The algorithm must recognize when the electrode is too close to a neuron and back away.

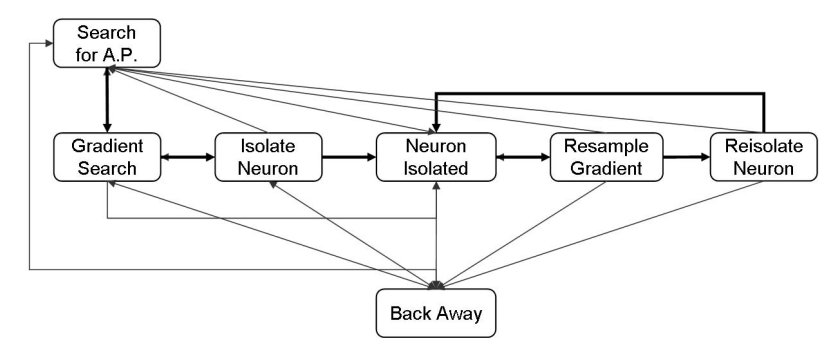

Fig. 3. Finite state machine for neuron isolation. All possible transitions are shown. The simplest path, which is discussed in the text, is shown by the thick arrows.

\section{Description of the Algorithm}

The basic architecture of the autonomous control algorithm consists of two layers. The inner layer is the stochastic optimization method developed in [10]. This method optimizes signal quality in the presence of action potentials, given that only noisy observations are available. The outer layer is a state machine, which performs the initial search for action potentials, monitors the cell isolation and maintenance processes, and commands appropriate actions to account for the biophysical challenges described above in section III.

\section{A. Stochastic Optimization}

Along a linear path of electrode travel past a neuron, plotting any metric of signal quality (e.g. peak-to-peak amplitude of the action potentials) forms a curve like that shown in Fig. 2, which we term an "isolation curve". The actual signal quality curve is not necessarily symmetric nor unimodal, but neither is required by the optimization method. The method is independent of the exact choice of signal quality function. The maximum of this curve corresponds to the closest approach of the electrode to the location on the neuron of action potential initiation. The optimization method seeks to position the electrode in the region of high signal quality. Observations of signal quality are measured at several locations and the underlying signal quality function is fit with a polynomial model. Bayesian probability theory is used to calculate the most likely model order to avoid overfitting. The electrode is then moved towards the maximum of the estimated signal quality function. More details of this procedure can be found in [10].

\section{B. Choice of Signal Quality Metric}

The quality of the action potential signal must be measured for two purposes: to measure the variation of signal quality around the neuron in order to find the optimal 
recording location (as described in the previous section), and to determine the acceptability of the current isolation (as described in the following section). There are several choices for each purpose.

1) Peak-to-Peak Amplitude (PTP): the average peak-topeak amplitude of the recorded action potentials.

2) Signal-to-Noise Ratio (SNR): the above metric normalized by the noise RMS voltage

$$
S N R=\frac{P T P}{\text { noise } R M S}
$$

3) Distance in Principal Components Space (DPCS):

Action potentials are projected onto their first two or three principal components and clustered (as shown in Fig. 1c and described in [10]). The signal quality metric is then any distance (Euclidean, Mahalanobis, etc.) between the mean of the cluster furthest from the origin and that of its nearest neighbor. If there is only one neuron present in the recording, its nearest neighbor will be the cluster corresponding to noise samples, and in this case DPCS will be similar to PTP.

4) Normalized Dist. in PC Space (NDPCS): the above metric normalized by the noise RMS voltage

$$
N D P C S=\frac{D P C S}{\text { noise } R M S}
$$

For constructing the isolation curve, PTP is the simplest choice. It is usually successful in isolating neurons and is the metric used to achieve the results in this paper. If there are several neurons of significant amplitude (as in Fig. 1), however, maximizing the peak-to-peak amplitude of one neuron may not 'separate' it from the other neuron, i.e. neither neuron will be isolated because action potentials cannot be reliably attributed to one of the neurons. DPCS is a metric which incorporates this notion of separation of multiple neurons. It may provide some improvement over PTP, but it is also sensitive to errors in the unsupervised clustering, particularly to the difficult task of determining the number of neurons present in a recording. Further attempts to implement this metric are future work.

For measuring the current quality of the isolation (for determining the proper state transition as described below), a normalized metric is used (SNR or NDPCS) to compare the signal quality to predetermined levels of isolation acceptability. The normalization accounts for variations in the overall signal level due to differences in the electrical characteristics between electrodes. Similarly to the above choice between PTP and DPCS, SNR is a simple and generally effective metric (which is used to achieve the results in this paper), while NDPCS may eventually offer improvement in the multiple neuron case if its sensitivity to errors in clustering can be reduced.

\section{State Machine}

A diagram of the supervisory control state machine is shown in Fig. 3. At each step, the algorithm samples the neural signal for a short length of time (typically 20 sec) and analyzes the signal. Depending on the analysis, the state machine may execute a change of state and/or send a move command to the microdrive to reposition the electrode.

A typical path through the state machine is as follows (the path described is highlighted in Fig. 3). The algorithm is usually started in the "Search for Action Potentials" state. It is assumed that the algorithm is started with the electrode initially and roughly placed in the region of neural tissue that is of interest for recording; an optional (and trivial) "Move to Target Depth" state can be used if this is not the case. In "Search for Action Potentials", the electrode is moved in steps of 30 microns until action potentials are detected and the state machine transitions to "Search for Gradient".

Action potentials are detected using the unsupervised wavelet-based detection method presented in [12]. Action potentials are considered to be present only when the frequency of events detected by the method exceeds a minimum firing rate, in this case $2 \mathrm{~Hz}$. Once detected, action potentials are aligned by their minimum and clustered using the finite mixture model method described in [10]. Clustering of the neuronal events helps to separate and identify the action potentials arising from distinct neurons. The signal quality of each cluster is calculated, and the current signal quality is taken to be the highest calculated.

In "Search for Gradient", the electrode is advanced in 15 micron steps, and signal samples are taken at each step. After the first few samples, observations are fit as described above in section IV-A. Bayesian probability is used to determine the most likely order of fit (details can be found in [10]). If the most likely order is higher than zero (i.e. at least a sloped line), then a statistically significant gradient has been found. If not, the electrode continues in steps of 15 microns until a gradient is detected.

When a statistically significant gradient is detected (and the signal quality is above a bottom threshold, below which the measurements of signal quality are unreliable), signaling that the electrode is on the isolation curve of a neuron, the state machine transitions to the "Isolate Neuron" state. This state performs the full optimization routine described above in section IV-A. A detailed description of the possible transitions out of this state is given below in section IV-D. If successful, the state machine transitions to "Neuron Isolated". In this state the electrode is held stationary and the signal is monitored.

Usually, the signal quality degrades as tissue drift causes the neuron to move away from the electrode tip. When the signal quality drops below $90 \%$ of its highest level, the algorithm attempts to reisolate the neuron. First, in the state "Resample for New Gradient", samples of signal quality are measured in 5 micron steps to determine the direction of neuron drift, and then in "Reisolate Neuron", the optimization technique described above is used again, hopefully leading once again to the state "Neuron Isolated". If, while in any state, the signal quality is above an upper threshold, the state machine transitions to "Back Away" and 




Fig. 4. Activity diagram for the state "Isolate Neuron". It begins at the solid circle on the left. Test junctions are shown by diamonds, test results are shown in brackets, and state transitions are shown by ringed circles with the name of the new state boxed.

the electrode is retracted 10 microns to avoid damaging the neuron.



Fig. 5. Thresholds of signal quality used to determine actions taken by the state machine. The left column shows the relative values of the thresholds. The right column shows the resulting interpretation and action made by the state machine. For example, for signal quality between MIN_SNR and STOP_SNR, stop moving and declare isolation if the top of the isolation curve is detected, otherwise keep moving to improve the isolation. If between STOP_SNR and MAX_SNR, however, it is not worth moving to improve the isolation and doing so may damage the neuron.

\section{State Machine Transition Logic}

The heart of the state machine is the logic which governs the transitions between states. To avoid unexpected behavior arising from an overly complex state machine, the simplest possible state machine was first tested. Its performance was monitored by experienced experimentalists. When the algorithm commanded a "non-optimal" electrode movement (i.e. a movement that an experienced experimentalist would not have commanded and did not lead to an isolation), an adjustment was made either to a parameter or statistical test in an existing transition, by adding a new transition to an existing state, or, rarely, by adding a new state.

As an example of the internal logic of the state machine and how it overcomes the biophysical challenges of isolation described above, the behavior of the algorithm while in the "Isolate Neuron" state will be described more fully here. The activity diagram of this state is shown in Fig. 4. Data is acquired and analyzed (action potentials are detected, aligned and clustered, signal quality is computed). Based on this analysis, the tests shown on the activity diagram are carried out to determine the state transition. The state transitions are principally determined by comparing the current signal quality with the thresholds in figure Fig. 5. As described in section IV-B, the signal quality metric used is $S N R$. The numerical values for the thresholds were chosen by consulting with experienced experimentalists and testing the algorithm with a range of values.

If no action potentials above a minimum threshold of signal quality (MIN_TRACK_SNR) are found, the variable Wait will be incremented and the self-transition is made to "Isolate Neuron". If this is the second step in a row in which no action potentials above MIN_TRACK_SNR have been found, the neuron being isolated is considered lost (it has drifted away, become inactive or has been damaged by the electrode) and the state machine returns to "Search for Action Potentials". Otherwise, the Wait variable is reset, and further tests are conducted to determine the new state. These tests principally compare the current signal quality $(S N R)$ with the predetermined thresholds. In addition to comparisons with the signal quality thresholds, there are two other tests on the activity diagram:

1) $\left(\left(\right.\right.$ move $\left.\left.<M I N \_M O V E\right) \&\left(S N R>M I N_{-} S N R\right)\right)$ : If the move command is lower than a predetermined threshold (move < MIN_MOVE), the top of the isolation curve has been reached. If the signal quality is acceptable $(S N R>$ 

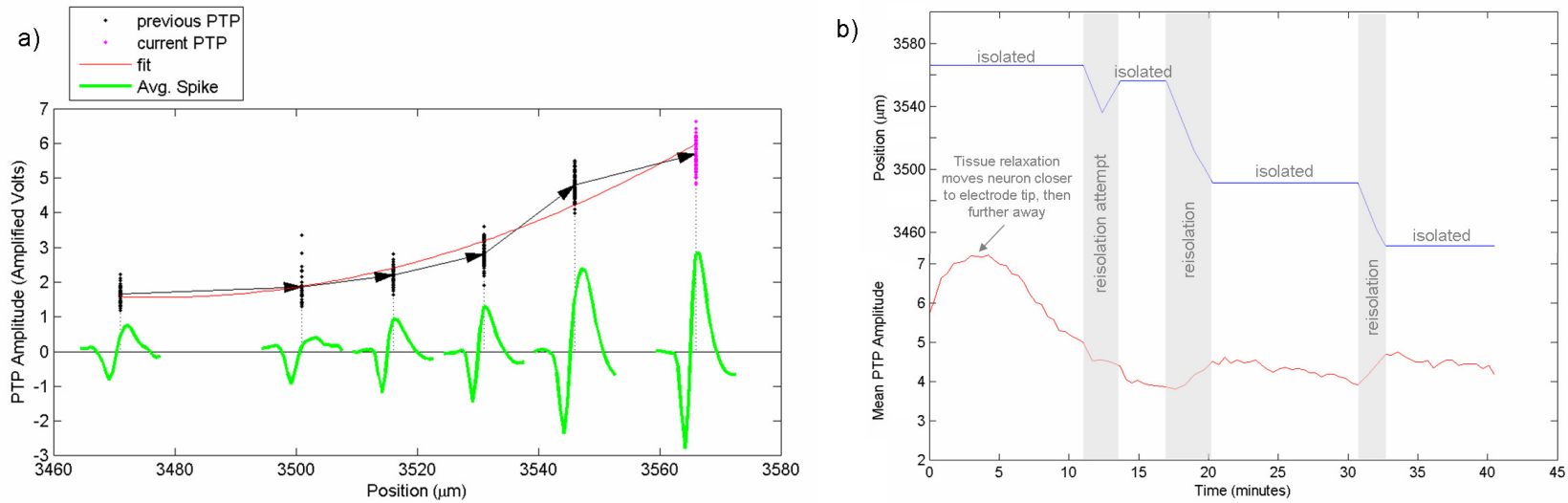

Fig. 6. Successful isolation of a neuron in macaque parietal cortex. a) shows the initial isolation. The observations of peak-to-peak amplitude at each position are shown in black dots with the final observation in magenta, the average action potential at each position in green, the reconstructed signal quality function in red, and the path of the electrode (always advancing in this example) by the black arrows. The leftmost observation is probably from another neuron, observed before detecting the isolation curve of the isolated neuron. The algorithm stopped at the rightmost position because the signal quality was high enough that further movement was unnecessary and may damage the neuron. b) shows the maintainance phase of the isolation. Both the elctrode position (blue) and the peak-to-peak amplitude (red) are plotted against time. Time zero is when the neuron is first isolated (the last observation shown on panel a). The signal improves and then degrades as the neuron drifts by the electrode. The nonhorizontal portions of the electrode position graph show periods where the state machine attempted to reisolate the neuron.

MIN_SNR), then the transition is made to "Neuron isolated". If not, then the neuron is deemed too far from the line of travel to be isolated, and the state machine returns to "Search for Action Potentials" to find a new neuron to isolate.

2) $(($ num_obs $\geq 5) \&($ Trend $\leq 0))$ : While in "Isolate Neuron", if the maximum of the isolation curve is not yet reached and the signal quality is not yet too high to fear neuron damage by continued movement, the self-transition to "Isolate Neuron" is typically made to continue the optimization. However, if after five observations (num_obs $\geq$ $5)$ the signal quality is showing a consistent downward trend (Trend $\leq 0$ ), then it is assumed neuron drift and/or electrode/tissue coupling are interfering with the isolation, and the fitted curve is unreliable. If the signal quality is acceptable, the transition is made to "Neuron Isolated", otherwise the state machine does not invest any more time attempting to isolate the current neuron and returns to "Search for Action Potentials" to find a new neuron to isolate.

\section{EXPERIMENTAL SETUP}

The algorithm has been implemented as the SpikeTrack Toolbox for MATLAB, available for download at

\section{http://robotics.caltech.edu/SpikeTrack}

The software can be used with any combination of microdrive (Thomas Recording $\mathrm{GmbH}$, FHC Inc., etc.) and data acquisition system (Plexon Inc, Tucker-Davis Technologies, etc.) by writing MATLAB M-files for moving the electrodes and acquiring the data streams. The most extensive testing of the algorithm has been performed using a single electrode FHC Inc. (Bowdoinham, ME) microdrive and a Plexon Inc. (Dallas, TX) data acquisition system, and it is this system for which results are shown. The algorithm is also being used in three other experimental setups, controlling a five-channel microdrive from Thomas Recording (Germany), a six-channel NAN drive (Plexon Inc.) and the three-channel microdrive described in [3]. These systems use a Plexon Inc. data acquisition system.

\section{RESULTS}

The algorithm has been used extensively to isolate and record dozens of neurons in the Posterior Parietal Cortex in rhesus macaque monkeys. Fig. 6 shows an example isolation. The initial isolation is shown in Fig. 6a. In Fig. 6b, the neuron drifts, first towards the electrode and then away, and is reisolated twice (the first reisolation attempt is unsuccessful due either to excessive drift or electrodetissue coupling). This is a relatively short isolation (it ended when the subject's behavior terminated the experiment), but clearly shows the effect of tissue drift and the success of the algorithm in maintaining good signal quality. Many neurons have been held isolated for several hours. The algorithm is currently being used successfully to isolate and hold neurons in several studies.

Controlled experiments to quantify the ability of the algorithm to isolate and maintain neurons, as compared to the ability of human experimentalists, are currently underway.

\section{CONCLUSION}

An algorithm has been described which autonomously isolates and maintains neural action potential recordings. The software implementation can, with minimal effort, be used to control electrodes in a wide variety of experimental setups. Future work will focus on improving the robustness of the algorithm. The algorithm shows less than optimal behavior in several challenging situations; such as when a neuron has been severely damaged and is releasing high amplitude but behaviorally irrelevant action potentials, and when multiple sporadically firing neurons are present 
in a recording. It is difficult for human experimentalists to achieve good isolations in these situations. Statistical tests and appropriate state transitions and electrode movements for these situations are currently being developed and tested. Also, as chronic repositionable arrays become available, the algorithm will be adapted for use in long term isolation of neurons, eventually recording high quality signal for years for use in clinical applications.

\section{ACKNOWLEDGMENT}

We thank Jorge Cham, Michael Wolf, and the members of the Andersen lab at Caltech, especially Bradley Greger, Bijan Pesaran, Kelsie Pesja and Nicole Sammons.

\section{REFERENCES}

[1] J. Cham, M. Wolf, R. Andersen, and J. Burdick, "Miniature Neural Interface Microdrive using Parylene-coated Layered Manufacturing," Proc. IEEE-RAS-EMBS Conf. on Biomedical Robotics and Biomechatronics, Pisa, Italy, Feb. 2006.

[2] E. Branchaud, J. Cham, Z. Nenadic, J. Burdick, and R. Andersen, "A Miniature Robot for Autonomous Single Neuron Recordings," IEEE Conf. on Robotics and Automation, Barcelona, Spain, April 2005.

[3] J. Cham, et.al. "Semi-chronic motorized microdrive and control algorithm for autonomously isolating and maintaining optimal extracellular action potentials", J. Neurophysiology, vol. 93, no. 1, pp. 570-579, January 2005 .
[4] S. Baker, et. al. "Multiple single unit recording in the cortex of monkeys using independently moveable microelectrodes," J. Neurosci. Methods, vol. 94 no. 1, pp. 5-17, Dec. 1999.

[5] I. Porada, I. Bondar, W. Spatz, and J. Kruger, "Rabbit and monkey visual cortex: more than a year of recording with up to 64 microelectrodes,” J. Neurosci. Methods, vol. 95, pp. 13-28, 2000.

[6] J. Williams, R. Rennaker, and D. Kipke, "Long-term neural recording characteristics of wire microelectrode arrays implanted in cerebral cortex," Brain Research Protocols, vol. 4, pp. 303-313, 1999.

[7] P. Rousche and R. Normann, "Chronic recording capability of the Utah intracortical electrode array in cat sensory cortex," J. Neurosci. Methods, vol. 82, pp. 1-15, 1998.

[8] C. Gray, P. Maldonado, M. Wilson, and B. McNaughton, "Tetrodes markedly improve the reliability and yield of multiple singleunit isolation from multi-unit recordings in cat striate cortex," J. Neurosci. Methods, vol. 63, pp. 43-54, 1995.

[9] C. Pang, J. Cham, Z. Nenadic, S. Musallam, Y. Tai, J. Burdick, and R. Andersen, "A New Multi-Site Probe Array with Monolithically Integrated Parylene Flexible Cable for a Neural Prosthesis", Proc. $27^{\text {th }}$ Conf. IEEE-EMBS, 2005.

[10] Z. Nenadic and J. Burdick, "A control algorithm for autonomous optimization of extracellular recordings", IEEE Trans. on Biomedical Eng., 2006. (in press)

[11] G. Buzsaki and D. Turner, "Dendritic properties of hippocampal CA1 pyramidal neurons in the rat: Intracellular staining in vivo and in vitro", J. Comp. Neurol., vol. 391, pp. 335-352, 1998.

[12] Z. Nenadic and J. Burdick. "Spike detection using the continuous wavelet transform," IEEE Trans. on Biomedical Eng., vol. 5, pp. 74-87, 2004. 